\title{
Alterstice
}

Revue internationale de la recherche interculturelle

International Journal of Intercultural Research

Revista International de la Investigacion Intercultural

\section{La Convention de Faro en perspective : analyse éthique du patrimoine culturel pour la société au Kosovo}

\section{Isabelle Brianso}

Volume 5, numéro 2, 2015

Patrimoine et interculturalité

URI : https://id.erudit.org/iderudit/1036688ar

DOI : https://doi.org/10.7202/1036688ar

Aller au sommaire du numéro

Éditeur(s)

Alterstice

ISSN

1923-919X (numérique)

Découvrir la revue

Citer cet article

Brianso, I. (2015). La Convention de Faro en perspective : analyse éthique du patrimoine culturel pour la société au Kosovo. Alterstice, 5(2), 21-32.

https://doi.org/10.7202/1036688ar

\section{Résumé de l'article}

Cet article tente d'analyser les enjeux éthiques, politiques et interculturels du patrimoine culturel européen pour la société au prisme de la Convention-cadre sur la valeur du patrimoine culturel pour la société (2005) dite « Convention de Faro ». Ce texte normatif du Conseil de l'Europe (CoE), innovant et original, prône un dessein politique et sociétal ancré dans une démocratie participative en faveur d'un patrimoine commun de l'Europe. La "Convention de Faro " s'inscrit donc en tant que réponse sociale et patrimoniale pour la société civile (communautés patrimoniales) par l'émergence de nouveaux modèles de gouvernance locale dans le respect d'un dialogue entre les nations membres et celles candidates à l'entrée dans l'Union européenne (Balkans). L'espace européen est confronté à des défis majeurs (environnement, patrimoine, société, crises) devant conduire les citoyens à une responsabilité individuelle et collective à l'égard du patrimoine fondée sur les principes démocratiques issus des droits de l'Homme. Enfin, nous analysons trois concepts fondateurs de deux textes normatifs majeurs (CoE, UNESCO) afin de comprendre les nouvelles modalités de l'enquête (terrain, méthodes) et la circulation des savoirs entre institutions pionnières en matière patrimoniale et les acteurs du territoire (top-down). L'étude sémantique de ces textes nous permettra d'appréhender les enjeux interculturels appliqués à un espace urbain et patrimonial au Kosovo (observatoire) et de mesurer en quoi il constitue un défi local de portée européenne.
Ce document est protégé par la loi sur le droit d'auteur. L'utilisation des services d’Érudit (y compris la reproduction) est assujettie à sa politique d'utilisation que vous pouvez consulter en ligne.

https://apropos.erudit.org/fr/usagers/politique-dutilisation/ 


\section{7}

alterstice

Revue Internationale

de la Recherche Interculturelle

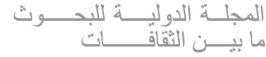

Revista International

de la Investigacion Intercultural

ARTICLE THÉMATIQUE

\section{La Convention de Faro en perspective : analyse éthique du patrimoine culturel pour la société au Kosovo}

Isabelle Brianso ${ }^{1}$

\section{Résumé}

Cet article tente d'analyser les enjeux éthiques, politiques et interculturels du patrimoine culturel européen pour la société au prisme de la Convention-cadre sur la valeur du patrimoine culturel pour la société (2005) dite "Convention de Faro ». Ce texte normatif du Conseil de l'Europe (CoE), innovant et original, prône un dessein politique et sociétal ancré dans une démocratie participative en faveur d'un patrimoine commun de l'Europe. La "Convention de Faro" s'inscrit donc en tant que réponse sociale et patrimoniale pour la société civile (communautés patrimoniales) par l'émergence de nouveaux modèles de gouvernance locale dans le respect d'un dialogue entre les nations membres et celles candidates à l'entrée dans l'Union européenne (Balkans). L'espace européen est confronté à des défis majeurs (environnement, patrimoine, société, crises) devant conduire les citoyens à une responsabilité individuelle et collective à l'égard du patrimoine fondée sur les principes démocratiques issus des droits de l'Homme. Enfin, nous analysons trois concepts fondateurs de deux textes normatifs majeurs (COE, UNESCO) afin de comprendre les nouvelles modalités de l'enquête (terrain, méthodes) et la circulation des savoirs entre institutions pionnières en matière patrimoniale et les acteurs du territoire (topdown). L'étude sémantique de ces textes nous permettra d'appréhender les enjeux interculturels appliqués à un espace urbain et patrimonial au Kosovo (observatoire) et de mesurer en quoi il constitue un défi local de portée européenne.

\section{Rattachement de l'auteure \\ ${ }^{1}$ Université d'Avignon et des Pays de Vaucluse, Centre Norbert Elias (EHESS, CNRS, UAPV, AMU), France}

\section{Correspondance}

isabelle.brianso@univ-avignon.fr

\section{Mots clés}

Conseil de l'Europe, patrimoine, interculturalité, UNESCO, Convention de Faro, Kosovo

\section{Pour citer cet article}

Brianso, I. (2016). La Convention de Faro en perspective : analyse éthique du patrimoine culturel pour la société au Kosovo. Alterstice, 5(2), 21-32. 


\section{Introduction}

Cet article vise à analyser la construction éthique, politique et interculturelle d'un texte de droit européen concernant les valeurs du patrimoine culturel pour la société. Ce texte normatif du Conseil de l'Europe (CoE), également dénommé "Convention de Faro", est entré en vigueur en 2011 après la ratification de l'État de Géorgie $^{1}$. Il s'inscrit dans un paradigme éthique innovant, car fondé sur la participation active des habitants et des "communautés patrimoniales" (art. 2), qui sont confrontés à des défis majeurs dans l'actualité écologique, politique, culturelle, économique et sociétale (conflits, crise économique, réchauffement climatique, crise identitaire, etc.). Ainsi, les valeurs du patrimoine pour la société s'apparentent désormais à un droit fondamental puisqu'elles " portent sur les aspects importants du patrimoine [... car] liées aux droits de l'homme, et promeuvent une conception plus large du patrimoine et de ses relations avec les communautés humaines, la société et la nation [...] » (Palmer, 2009). Dans ce contexte, le Conseil de l'Europe tente depuis 2011 de mettre en place un dialogue démocratique local en interaction politique avec la société civile (participation citoyenne) en vue de construire un "patrimoine commun de l'Europe " (art. 3) pour la société, basé sur deux principes fondateurs européens et interculturels: "diversité » et "unité ». Cet idéal démocratique fondé sur un partage de valeurs culturelles communes, défendu dès la fin de la Seconde Guerre mondiale par les grandes institutions internationales onusiennes, dont I'UNESCO, connaît néanmoins des freins pluriels quant à sa mise en œuvre. Nous proposons d'étudier trois concepts fondateurs qui articulent l'architecture sémantique de ce standard européen puis de les mettre en perspective avec trois concepts analogues de la Convention du patrimoine mondial de l'UNESCO (1972). Ce choix comparatif (COE, UNESCO) porte sur l'observation de nouvelles postures éthiques en matière de patrimoine culturel en raison de l'évolution de la notion de " patrimoine(s) " selon les organisations internationales (UNESCO, CoE) ainsi qu'en raison de l'émergence de nouvelles stratégies d'acteurs du territoire ("peuples » ou "communautés patrimoniales») à partir d'articulation déséquilibrée d'échelles, allant du local (ville) à l'international (Europe, monde). Par ailleurs, l'analyse de ces concepts au prisme d'une approche interculturelle vise à s'interroger sur les positionnements éthiques de deux institutions pionnières en matière de patrimoine culturel ainsi que sur les écueils relatifs à leur mise en œuvre locale, et vise par conséquent à se demander en quoi un texte normatif européen peut-il être porteur (ou non) de sens auprès des citoyens et des populations locales. Sur quels fondements éthiques et interculturels repose-t-il? Vers quelle(s) construction(s) patrimoniale(s) européenne(s) souhaite-t-il s'orienter? Autant de questionnements complexes à la source de l'analyse des mécanismes internationaux de patrimonialisation, au croisement disciplinaire des sciences humaines et sociales. À partir de ce positionnement réflexif, nous appliquons notre approche méthodologique à un territoire localisé au sud-est de l'espace politique européen, le Kosovo. En effet, cette république des Balkans a connu des tensions et des déconstructions patrimoniales dans son histoire contemporaine, ce qui a conduit le CoE à mener une étude ${ }^{2}$ expérimentale, par l'enquête en tant qu'observatoire in situ des principes de la Convention de Faro. Précisons que cette étude de cas reste marginale et ne peut prétendre à être un " modèle Faro " pour les autres pays européens en raison du contexte géopolitique singulier qui caractérise ce territoire ayant subi un conflit armé. Néanmoins, nous essaierons de comprendre en quoi les enjeux interculturels appliqués à un espace urbain et patrimonial complexe, celui de la ville de Junik (située non loin de la capitale kosovare, Pristina), constituent un défi local de portée européenne.

\section{La Convention de Faro, un cadre européen à la notion de patrimoine}

\section{Le cadre éthique du Conseil de l'Europe : une "démocratie » patrimoniale et citoyenne}

Le CoE est une organisation intergouvernementale (États membres) créée au lendemain de la Seconde Guerre mondiale par un traité international, le traité de Londres, le 5 mai 1949 (Conseil de l'Europe, 1949). Sans présenter la mécanique bureaucratique de cette organisation européenne de premier plan en matière de démocratie, il est légitime de rappeler que sa construction idéologique, morale et politique repose sur un texte fondateur, la

\footnotetext{
${ }^{1} 4$ février 2011 - dixième signature obligatoire pour l'entrée en vigueur de la Convention-cadre.

${ }^{2}$ L'auteure de l'article a mené cette enquête qualitative (2014-2015) au Kosovo en tant qu'experte (Marie Curie Fellow, IEF, FP7) pour le CoE. Cette étude a été mise en œuvre dans le cadre du programme-joint "Support à la promotion de la diversité culturelle au Kosovo » (2009-2015) et coordonnée par la Direction de la gouvernance démocratique (DG II, CoE) en collaboration avec le Bureau CoE de Pristina au Kosovo.
} 
Convention européenne des droits de l'homme (Conseil de l'Europe, 1950). Au lendemain de la Seconde Guerre mondiale, l'Europe peine à se reconstruire tant sur le plan social et économique que politique, à la suite de l'occupation allemande (1939-1945), des destructions massives de ses territoires d'ouest en est et de la Shoah. Elle est dès lors confrontée à de nouveaux défis politiques, moraux et démocratiques de réconciliation entre les peuples en vue de transmettre aux générations futures une Europe commune fondée sur la paix des nations, garante d'un patrimoine commun à sauvegarder et d'une solidarité éthique basée sur des valeurs partagées entre ces nations. Cette posture démocratique pose les fondements d'une construction patrimoniale ancrée dans une gouvernance politique chargée à la fois de fédérer les peuples européens (unité) et de leur garantir une pérennité juridique, favorable à la diversification culturelle et sociale des acteurs qui habitent cet espace géopolitique en reconstruction. Ainsi, le principe de "diversité » - marqué principalement par la géographie, le multilinguisme, I'histoire et les nouvelles dynamiques sociétales - fixe rapidement les jalons politiques et culturels d'une Europe interculturelle, tant par ses différences que ses ressemblances, car « les dynamiques interculturelles européennes se déploient également entre les différentes formes de sociétés qui ont façonné les pays européens au cours des siècles " (Demorgon, Müller et Lipiansky, 2003, p. 1). S'ouvre alors un dialogue interculturel dès l'entrée des premiers États membres au sein de l'Union européenne, à partir de programmes phares encourageant des actions communes (Journées européennes du patrimoine, Nuit européenne des musées) devant susciter des coopérations et des synergies culturelles dans l'espace européen (labels "Capitale européenne de la culture », "Itinéraires culturels »). Cette vision d'une politique culturelle commune va néanmoins faire émerger divers déséquilibres entre une gouvernance normée en matière patrimoniale (conventions, traités, déclarations) et des jeux d'acteurs locaux revendiquant leur identité locale, nationale et régionale. Notons que le CoE a adopté et ratifié pas moins de sept ${ }^{3}$ conventions "culture » en plus de soixante ans d'existence (entre 1954 et 2005), dont les discours ont porté principalement sur la protection et la sauvegarde de l'objet en tant que patrimoine culturel européen (archéologie, architecture, cinéma), à l'exception du dernier texte normatif adopté puis ratifié par le CoE, davantage centré sur les valeurs du patrimoine culturel pour la société. II est intéressant de souligner que la Convention-cadre sur la valeur du patrimoine culturel pour la société (Conseil de l’Europe, 2005), dénommée dans cet article Convention de Faro, est présentée en tant que réponse politique en faveur de la société européenne, dont le dessein est de débattre et de fabriquer un "vivre ensemble » citoyen à partir de nouveaux modèles de développement fondés sur plus de participation citoyenne. Cette convention s'inscrit donc au cœur du construit discursif en matière de " démocratie citoyenne » comme moteur politique, social et éthique entre les peuples européens, tout en pointant une véritable complexité conceptuelle (concepts fondateurs) au prisme d'articulation d'échelles délicate à mettre en œuvre, allant de l’international (Europe) au local (territoire, ville).

\section{Trois concepts fondateurs de la Convention de Faro : communauté patrimoniale (art. 2), patrimoine (art. 2), patrimoine commun de l'Europe (art. 3)}

Comme nous l'avons vu, la Convention de Faro est un outil normatif qui vient compléter une pluralité d'instruments internationaux majoritairement onusiens (UNESCO) déjà actifs à l'échelle mondiale - citons la Convention du patrimoine mondial (1972), la Convention sur la sauvegarde du patrimoine culturel immatériel (2003) et la Convention sur la protection et la promotion de la diversité des expressions culturelles (2005). Néanmoins, l'originalité de ce texte normatif européen repose sur sa nature, car pour la première fois c'est l'individu qui est reconnu comme acteur légitime de l'action patrimoniale, et non l'objet patrimonial (monument, collections, etc.). Ce postulat n'est cependant pas à confondre avec les courants théoriques issus d'une approche communicationnelle de la culture et de ses visiteurs (musée, site historique, bibliothèque, etc.), bien que certains rapprochements réflexifs puissent être faits, notamment par l'étude des pratiques et des représentations à l'ère d'un tourisme mondialisé. D'après ce texte, les individus sont au cœur du processus en tant que " communauté patrimoniale » dotée d'une responsabilité individuelle et collective envers ce patrimoine (article 1). Ainsi, Liévaux (2009, p. 49) définit le concept de «communauté patrimoniale » en tant que regroupement "variable d'individus

\footnotetext{
${ }^{3}$ Convention culturelle européenne (1954); Convention européenne pour la protection du patrimoine archéologique (1969); Convention européenne pour des infractions visant des biens culturels (1985); Convention pour la sauvegarde du patrimoine architectural de l'Europe (1985); Convention européenne pour la protection du patrimoine archéologique (révisée) (1992); Convention européenne pour la coproduction cinématographique (1992); Convention-cadre du Conseil de l'Europe sur la valeur du patrimoine culturel pour la société (2005).
} 
et [qui] permet d'associer pleinement les citoyens à la construction du patrimoine commun de l'Europe ". Dans ce contexte, l'implication réelle de la société civile dans les processus de patrimonialisation devient « une condition essentielle de la diversité du patrimoine culturel » (Liévaux, 2009) et à la construction collective de la participation démocratique (art. 12). Si cette construction politique du patrimoine de l'espace européen semble convenir au CoE, d'autres concepts restent néanmoins à préciser, et en premier lieu celui de "patrimoine ": bien que récurrent dans la Convention de Faro, ce dernier n'est pas véritablement défini, puisqu'il " constitue un ensemble de ressources héritées du passé et que les personnes considèrent [...] comme un reflet et une expression de leurs valeurs, croyances, savoirs et tradition en continuelle évolution » (art. 2), définition très large qui fait potentiellement de tout type de " ressource » un patrimoine, sans pour autant circonscrire l'objet patrimonial - au contraire, c'est une ouverture à toutes formes et expressions culturelles et naturelles en tant qu'approches intégrées pouvant « renforcer la cohésion sociale en favorisant le sentiment de responsabilité partagée envers l'espace de vie commun » (art. 8). Cette polysémie patrimoniale, sans frontière, marque une rupture dans l'analyse normative de l'objet patrimonial, car il n'y a plus de contrainte à des critères d'évaluation pour sa définition, comme le stipule I'UNESCO, mais celle-ci repose sur sa seule reconnaissance par la société. Cette originalité de forme n'est pas sans provoquer questionnements et débats, puisque la société civile devient seule juge de ce qui fait ou non patrimoine. Ainsi, l'interprétation de ces deux concepts fondateurs est sujette au libre arbitre des acteurs impliqués dans leur mise en œuvre locale (États membres, société civile) qui, pour certains, y voient plutôt un levier intellectuel et juridique à l'accès aux standards de l'Union européenne en faveur de pays candidats (Europe du Sud-Est) comme mécanique politique d'accélération du processus d'intégration (Filipovic, 2009). En effet, Filipovic (2009) précise que la ratification des conventions du CoE par les pays tiers à l'Union européenne (Balkans, ex-pays soviétiques) «[...] accélérera le processus d'intégration, qu'elle permettra de se mettre à I'unisson des tendances courantes, d'obtenir plus facilement des informations et d'accéder à différentes sources de financements ainsi qu'à l'assistance d'experts [...] " (Filipovic, 2009, p. 51). Enfin, la " Convention de Faro » met en avant un concept transversal relevant d'une histoire culturelle commune garante d'un " patrimoine commun de l'Europe » (article 3) en tant que ressource partagée par l'ensemble des citoyens dans le temps et l'espace, structurée à partir des « idéaux, des principes et des valeurs, issus de l'expérience des progrès et des conflits passés [...] » (art. 3). Si ce concept est très difficile à définir, à l'instar des deux précédents (" communauté patrimoniale » et " patrimoine »), il est néanmoins éminemment politique et géostratégique à l'heure des crises identitaires nationales et régionales sur fond de tensions économiques et de pourparlers asymétriques dans une Europe élargie. Sans dresser une liste exhaustive des concepts fondateurs de la Convention de Faro, les trois concepts cités précédemment structurent le texte normatif de la Convention de Faro en tenant compte des enjeux locaux de construction (" patrimoines, individus, Europe ») devant tendre vers une démocratie participative idéalement partagée sur l'ensemble du territoire européen et au-delà (Russie).

\section{Nouvelles postures du patrimoine culturel au défi de l'interculturel : Conseil de l'Europe et UNESCO}

\section{Convention de Faro et Convention du patrimoine mondial, une mise en perspective du défi interculturel et identitaire}

Comme nous l'avons précisé, le CoE et I'UNESCO sont les fruits de réflexions politiques au lendemain de la Seconde Guerre mondiale, dont les enjeux éthiques reposent sur le dialogue entre les nations. L'UNESCO, reconnue en tant qu'organisation interculturelle composée d'États membres seuls représentants politiques de nations singulières, collabore activement à la mise en œuvre de programmes et de projets issus de la diplomatie culturelle (paix). La diplomatie joue donc un rôle central dans ces échanges internationaux et interculturels mis en place par I'UNESCO, car elle favorise les relations et les rencontres entre les nations pour maintenir la paix et la sécurité « [...] à condition qu'elle soit équilibrée par une autre perspective qui aborde plus résolument, profondément et obstinément les réalités difficiles, voire conflictuelles. [...]" (Demorgon, 1989, p. 65-66). Le premier grand programme de I'UNESCO en matière d'interculturalité fut le Projet majeur relatif à l'appréciation mutuelle des valeurs culturelles de l'Orient et de l'Occident. Ce projet interdisciplinaire, mieux connu sous le nom de « Projet majeur Orient-Occident ", a duré dix ans (1956-1966) et il a ouvert le dialogue interculturel en tant que rencontre exploratoire (Demorgon, 1989) entre les nations par des activités basées sur les échanges culturels entre les différentes régions du monde, régions pour lesquelles l'éducation auprès des plus jeunes (écoles primaires) constitue la principale articulation « pour la compréhension et la coopération internationale ». La mise en œuvre de ce projet ambitieux et complexe par les sujets traités en matière de rencontre culturelle entre les peuples

Alterstice - Revue Internationale de la Recherche Interculturelle, vol. $5, n^{\circ} 2$ 
reflète une véritable volonté politique de la part de l'UNESCO à favoriser un rapprochement des cultures tout en se confrontant à de réelles difficultés de terrain, elles-mêmes culturelles, mais aussi administratives et politiques, amenant les experts à reconnaître la difficulté " de préciser et de mesurer l'apport propre du projet " (Demorgon, 1989), bien que de véritables résultats aient pu être avérés. La Conférence générale de 1966 valide les dix années du projet comme " une période exploratoire, pendant laquelle on n'a fait qu'aborder, sans plus, un problème très complexe " (UNESCO, 1968). Ce projet, bien qu'ancien, souligne la volonté des organisations internationales d'instaurer un dialogue entre les cultures, mais aussi les difficultés des enjeux interculturels appliqués à la culture, aux sociétés et au patrimoine. Au tournant des années 1970, la Convention du patrimoine mondial (UNESCO, 1972) s'inscrit dans ce prolongement politique à vocation écologique et de dialogue entre les nations dans la mesure où elle marie à l'échelle planétaire deux types de biens (culture, nature) en apparence antagonistes (Batisse et Bolla, 2003) devant former un tout universel. L'analyse discursive simple par occurrences de nos deux textes normatifs (UNESCO, CoE) montre que la notion d'interculturalité est suggérée mais n'est jamais mentionnée. Pourtant, l'émergence de la notion d'interculturalité est bien l'aboutissement de discussions sous tension au sein de I'UNESCO, à laquelle on associe communément l'équivalence de " dialogue entre les cultures " comme le souligne Clanet (1990, p. 24) lors de la Conférence générale de I'UNESCO (1976) : "À côté du principe d'authenticité culturelle, il convient de poser le concept de dialogue entre les cultures. Sous peine de favoriser les cloisonnements nationaux et le sectarisme sous des formes diverses, il importe d'ouvrir chaque culture à toutes les autres dans une perspective largement internationale. »

Le Conseil de l'Europe promeut à son tour le " dialogue » appliqué au patrimoine culturel (art. 7) " dans le respect de la diversité des interprétations ». Ainsi, l'usage récurrent ou suggéré du concept d'interculturalité dans les liens à l'Autre et entre cultures différentes (Ladmiral et Lipiansky, 1989) au sein de ces deux institutions pionnières nous permet de situer notre positionnement quant au choix d'une définition de l'interculturalité dans cet article: " ensemble de processus - psychiques, relationnels, groupaux, institutionnels, etc. - générés par les interactions de culture, dans un rapport d'échanges réciproques et dans une perspective de sauvegarde d'une relative identité culturelle entre des partenaires en relation » (Clanet, 1990 p. 21). Les deux conventions (label international et label "Faro ») s'appuient sur des éléments identitaires très normés et une relative ambiguïté interprétative : un patrimoine commun ou universel (mémoire ou ressources) partagé par les peuples (UNESCO) et les communautés patrimoniales (CoE) issues d'approches stratégiques (gouvernance) singulières (sécurité/paix ou démocratie participative) que nous pouvons résumer comme suit (tableau 1) :

Tableau 1 : Éléments identitaires des deux textes normatifs (UNESCO, CoE)

\begin{tabular}{|l|l|l|}
\hline & \multicolumn{1}{|c|}{ UNESCO CoE } \\
\hline Norme/Standard & $\begin{array}{l}\text { Label international de protection du } \\
\text { patrimoine mondial }\end{array}$ & $\begin{array}{l}\text { Label "Faro » de démocratie } \\
\text { participative }\end{array}$ \\
\hline Patrimoine (universel/commun) & $\begin{array}{l}\text { Mémoire du monde } \\
\text { (bien culturel/bien naturel) }\end{array}$ & $\begin{array}{l}\text { Ressources patrimoniales } \\
\text { (valeur du patrimoine) }\end{array}$ \\
\hline Société & Peuples du monde & $\begin{array}{l}\text { Communautés patrimoniales dans } \\
\text { l'Europe élargie }\end{array}$ \\
\hline Gouvernance & Paix/sécurité & Démocratie \\
\hline
\end{tabular}

Notons que la notion de " patrimoine(s) » ayant fait l'objet de nombreuses occurrences dans notre corpus d'études - ce que nous présentons dans le tableau 2 -, il convient de la préciser à partir des points de vue des organisations (UNESCO, CoE) afin de mieux appréhender les dynamiques d'échelle (locale ou globale).

\section{Du concept à l'interprétation : retour sur des dynamiques d'échelle de la notion de patrimoine}

Selon les deux organisations (UNESCO, CoE), la notion de patrimoine est soit structurée et normée par la matérialité (monument, architecture, site) puis évaluée par des experts (ICOMOS, UICN), soit polysémique et apparentée aux ressources collectives héritées du passé par les individus (CoE). Pourtant, le caractère d'universalité patrimoniale est bien présent dans les deux textes, sauf qu'il est conçu à partir d'articulation d'échelles bien 
distinctes. Les caractères " universel » (UNESCO) et " commun » (COE) des valeurs patrimoniales auxquelles sont associés les biens ne diffèrent pas tant d'un point de vue éthique que d'un point de vue géographique. Ainsi, le CoE délimite l'espace géopolitique européen comme seule aire patrimoniale légitime permettant la construction d'une identité culturelle commune des peuples européens en tant qu'espace physique (territoires) et symbolique (histoire, échanges). À l'inverse, l'UNESCO considère à partir de critères établis par le Comité du patrimoine mondial (bien culturel, bien naturel) que la valeur universelle exceptionnelle (Labadi, 2013) doit être démontrée par les acteurs locaux et perçue comme telle par les groupes d'experts afin d'ériger un site au rang de " patrimoine commun de l'humanité " (Chenevet et Novello-Paglianti, 2015). Cette logique d'approche s'inscrit donc dans les fondements politiques de ces deux organisations - en effet, l'interprétation de la notion de patrimoine par I'UNESCO et le CoE résulte de postures patrimoniales différentes. Comme nous l'avons évoqué dans la section précédente, le CoE organise son discours à partir d'une "démocratie citoyenne" portée par les sociétés européennes d'ouest en est (Russie incluse). Les citoyens ou les individus répondent donc à des logiques d'acteurs sur les territoires nationaux et régionaux (les Balkans par exemple) pour la mise en œuvre collective de la Convention de Faro à l'échelle locale. Le CoE favorise une logique du consensus en matière patrimoniale, au sens où les acteurs locaux doivent s'approprier collectivement l'objet patrimonial à préserver puis agir à partir des principes qui découlent de la convention au sein des communautés patrimoniales des territoires concernés. Il n’y a donc pas de critère sur les valeurs du patrimoine à l'instar de l'UNESCO, mais un consensus collectif de ce qui fait patrimoine pour la société et les communautés patrimoniales (ONG, associations, etc.). Cette approche (patrimoine, communauté, universalité) déroute les acteurs locaux en quête de référentiel (critère, norme) malgré certaines orientations et indications données en matière de préservation et de protection du patrimoine culturel européen. À titre d'exemple, le Plan régional du patrimoine ${ }^{4}$ (2012-2015) du Kosovo Ouest proposé par le CoE applique la notion de patrimoine à l'ensemble des éléments qui ont une valeur patrimoniale selon les indications de Faro - la liste est longue : monument, archéologie, objets, patrimoine architectural, faune, flore, paysage, géologie, jardins, parcs, etc. L'approche européenne du patrimoine s'apparente au courant systémique issu des courants écologiques (Matagne, 2009) en tant que gestion intégrée des éléments patrimoniaux, à l'instar d'un " écosystème " local de portée européenne. Ainsi, la lecture attentive des deux conventions traitées à partir d'un outil lexicométrique simple (occurrences) souligne l'identité nominale de la notion de "patrimoine » des deux textes normatifs. Nous constatons que la Convention de Faro est marquée par la dualité du nombre (pluriel/singulier) et la diversité (architecture, archéologie, communautés, jeunes) alors que la Convention du patrimoine mondial de l'UNESCO se caractérise par l'universalité du nombre (singulier), ce que nous présentons de manière synthétisée dans le tableau 2.

\section{Tableau 2 : Les occurrences de la notion de " patrimoine " du corpus d'études (CoE, UNESCO)}

\begin{tabular}{|c|c|c|c|}
\hline \multicolumn{2}{|l|}{ CoE } & \multicolumn{2}{|l|}{ UNESCO } \\
\hline «Convention de Faro » & Occurrences & $\begin{array}{l}\text { "Convention } d u \quad \text { patrimoine } \\
\text { mondial " }\end{array}$ & Occurrences \\
\hline Patrimoine(s) & 74 & Patrimoine & 78 \\
\hline $\begin{array}{l}\text { "Patrimoine(s) » suivi d’un adjectif } \\
\text { (culturel, architectural, } \\
\text { archéologique, [des] communautés, } \\
\text { [des] jeunes) }\end{array}$ & 58 & $\begin{array}{l}\text { «Patrimoine » suivi d'un adjectif } \\
\text { (culturel, naturel) }\end{array}$ & 42 \\
\hline "Patrimoine commun de l'Europe » & 5 & «Patrimoine mondial » & 30 \\
\hline
\end{tabular}

Note : Ces occurrences sont obtenues à partir d'un traitement simple avec le logiciel Lexico 3.

La présentation des occurrences renforce le caractère comparable des deux conventions, notamment concernant le mot " patrimoine(s) » et ce qui le qualifie (adjectif). II existe bien un certain équilibre sémantique dans la construction lexicale de ces standards internationaux, bien que I'UNESCO précise clairement ce qui spécifie le " patrimoine mondial ", à savoir la nature et la culture selon les dix critères établis par le Comité du patrimoine mondial. À l'inverse, les occurrences relevées et appliquées au " patrimoine commun de l'Europe » suggèrent une difficulté quant à l'identité réelle d'un patrimoine commun partagé sur l'ensemble de l'espace européen. L'analyse

\footnotetext{
${ }^{4}$ Regional Heritage Plan for Kosovo West (2012-2015).
} 
lexicométrique simple de la Convention de Faro fournit un nombre très faible ( 5 occurrences) de répétitions de l'appellation de "patrimoine commun de l'Europe ", ce qui corrobore l'interprétation locale du texte européen par la société civile - par ailleurs, notons que le terme "Europe " est essentiellement associé aux organisations européennes et non à l'espace géographique.

Nous pouvons conclure que l'analyse éthique de trois concepts fondateurs de la Convention de Faro au prisme de l'interculturalité suscite des interrogations quant aux applications réelles de cet outil normatif sur un territoire donné (Europe) et quant à la manière dont les acteurs locaux interprètent et mettent en œuvre cet instrument complexe. Nous avons ainsi voulu vérifier la validité de cette posture éthique aux Balkans (Kosovo), en raison de la création d'un observatoire expérimental de mise en œuvre des principes de la démocratie participative depuis 2011. Nous précisons que, même si cet espace géographique pilote présente de réelles perspectives d'adhésion aux principes de Faro, cette étude de cas reste exploratoire, sur un territoire en reconstruction.

\section{La Convention de Faro en Europe du Sud-Est : enjeux politiques et interculturels}

\section{Un instrument européen ambitieux doté d'une mise en œuvre déséquilibrée}

La Convention de Faro a retenu l'attention de certains pays d'Europe du Sud-Est car elle offre une formidable opportunité politique d'intégration européenne grâce à l'adhésion (ratification) aux " principes de Faro " fondés sur la responsabilité collective et individuelle en matière de patrimoine culturel, l'amélioration de la qualité de la vie, la construction de sociétés pacifiques et démocratiques, etc. Le Programme régional pour le patrimoine culturel et naturel dans le sud-est de l'Europe (RPSEE), lancé en 2003, a largement contribué à la signature puis à la ratification de la Convention de Faro par les pays de l'ex-Union soviétique et des Balkans, que ce soit l'Albanie, la Bosnie-Herzégovine, la Bulgarie, la Croatie, le Monténégro, la Serbie ou la République du Kosovo. Ces pays ont donné une véritable impulsion à l'entrée en vigueur en 2011 de la Convention de Faro, grâce à l'appui du Conseil de l'Europe dans la région, en fédérant et en installant un dialogue politique entre ces territoires. Les diversités culturelles, religieuses, ethniques et politiques qui, au cours des années 1990, étaient à l'origine de tensions et de conflits, ont su être transformées en ressources pour un "développement local faisant des habitants les acteurs principaux de la transformation du territoire et assurant une participation démocratique dans les projets » (Conseil de l'Europe, 2009, p. 56). Les dynamiques et les synergies suscitées en Europe du Sud-Est ont ainsi incité les instances européennes à renforcer les programmes régionaux de coopération en matière de patrimoine et de diversité culturelle, par exemple le programme joint (COE/UE) à la promotion de la diversité culturelle au Kosovo (PCDK). Ces initiatives ont par ailleurs pointé des difficultés d'ordre structurel liées aux administrations et municipalités locales qui, pour la plupart, ont découvert en même temps qu'elles ratifiaient la Convention de Faro les obligations européennes dont elles devaient désormais s'acquitter. De même, la participation démocratique tant revendiquée dans la Convention de Faro a dû s'organiser localement afin de répondre à des changements sociétaux profonds, dans le but de tirer tous les bénéfices pour les individus, la société et les organisations patrimoniales, comme le souligne Sharon Goddard (2009, p. 153). Ces bénéfices, dont aucun acteur de la gouvernance ne doute pour la société, semblent plus complexes quant à leur mise en œuvre pérenne et durable auprès de sociétés en mutation et en reconstruction. Précisons par exemple quelques asymétries soulignées par certains auteurs quant au processus de gouvernance des acteurs d'Europe du Sud-Est :

- Politique de partenariats asymétrique: Sharon Goddard (2009, p. 157) souligne la nécessité d'établir des partenariats efficaces en vue de construire une vision stratégique commune. Néanmoins, ces partenariats se résument le plus souvent à des recherches de fonds auprès d'organisations internationales dont les véritables bénéficiaires restent majoritairement les acteurs locaux déjà organisés en administration (municipalités, ministères, etc.) ou en institutions habilitées à gérer des fonds européens et internationaux (ONG, etc.);

- Principes de Faro déséquilibrés entre experts et acteurs locaux: de nombreux auteurs (Bortolotto, 2012; Brianso et Girault, 2014; Paquette, 2012) soulignent les déséquilibres récurrents entre experts habilités à évaluer par critères le patrimoine culturel et les acteurs locaux habilités à appliquer in situ les principes des dites conventions;

- Une participation démocratique idéalisée : Chenevet et Novello-Paglianti (2015), Brianso et Girault (2014) et Liévaux (2009) soulignent l'originalité de la participation démocratique en tant qu'innovation éthique en 
matière de patrimoine culturel. Néanmoins, cette dernière reste trop souvent idéalisée, limitée au champ d'universalité prôné par les grands textes fondateurs liés aux droits de l'Homme. Cette utopie patrimoniale est sujette à débat quant à sa mise en œuvre réelle sur le plan local par les instances internationales - ces dernières ayant tendance à soutenir les initiatives locales plus qu'elles n'agissent en lien direct avec les acteurs du territoire.

Cette gouvernance asymétrique a donc conduit le CoE à réorganiser les résultats de terrain liés à la mise en œuvre des textes normatifs par la création d'observatoires et réseaux thématiques (Observatoire HEREIN) ou géographiques (réseau LDPP ${ }^{5}$ ), dont la mission principale serait de fédérer les acteurs partenaires et maîtriser la communication. Il est à noter que le développement récurrent de ces instruments (observatoire, réseau, laboratoire) par projets ${ }^{6}$ tend à remettre en question la place de l'enquête (quantitative, qualitative), ses méthodes et les résultats produits au sein du Conseil de l'Europe, comme cela a déjà pu être analysé par Anne Piponnier (2012a, 2012b, 2014) dans le cadre de ses travaux liés à l'émergence d'observatoires dans les équipes de recherche. Elle précise que « faire le choix de restituer les données et le travail de l'enquête dans un observatoire consiste en effet pour les acteurs, non seulement à concevoir un dispositif d'accueil pour la recherche, mais c'est aussi accepter [...] de prendre en charge la portée communicationnelle de ce dispositif qui crée un horizon d'attentes, non seulement scientifiques, mais également sociales et économiques » (Piponnier, 2014, p. 16).

\section{Le Kosovo : un observatoire patrimonial in situ du Conseil de l'Europe}

La République du Kosovo a fait l'objet d'une attention particulière de la part de la communauté européenne en raison des tensions et des conflits dans cette région des Balkans au début des années 1990. Territoire sensible par sa localisation géostratégique entre l'Europe du Sud-Est, la péninsule balkanique et l'ex-Empire ottoman, le Kosovo constitue un carrefour d'échanges culturel, politique, religieux et ethnique. Ce petit territoire (10 $\left.908 \mathrm{~km}^{2}\right)$ enclavé entre la Serbie, le Monténégro, l'Albanie et la Macédoine a connu de nombreuses ruptures politiques dans son histoire contemporaine (invasions, guerres, conflits). Citons les Guerres balkaniques de 1912 et de 1913, ou plus récemment les conflits interethniques lors de la chute de l'ex-Yougoslavie, qui conduira le Kosovo à proclamer son indépendance le 17 février 2008 grâce au soutien de la communauté européenne et internationale. Malgré les tensions, le Kosovo a su maintenir une stabilité politique dans la région et collaborer à un dialogue interreligieux (90\% de la population est musulmane), interethnique ( $88 \%$ d'Albanais, $7 \%$ de Serbes) et interculturel (langues, traditions, identités). Ce contexte politico-culturel séculaire très complexe, que nous ne développerons pas dans cet article, a constitué un observatoire expérimental pour le CoE quant à la mise en œuvre des principes de Faro sur ce territoire (De Sède-Marceau et Moine, 2012). Notons que le Plan d'Action pour la Promotion de la Convention-Cadre de Faro (adopté en novembre 2014) précise les axes prioritaires ${ }^{7}$ retenus à la promotion et à la mise en œuvre de ce texte normatif ainsi que les principes ${ }^{8}$ issus des réflexions du Forum de Marseille (2013), dont

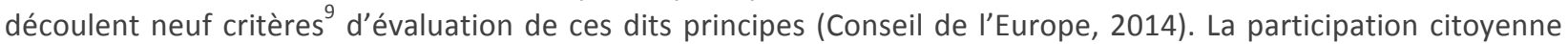
s'inscrit donc en tant qu'engrènement politique majeur à mouvoir sur le territoire kosovar, bien que devant répondre à deux questions cruciales : quelle(s) forme(s) prend-elle et comment la participation démocratique se dessine-t-elle à l'échelle locale? En effet, il n'existe ni guide, ni méthodologie, ni véritable outil technique à l'usage des acteurs qui rende lisibles les modalités de mise en œuvre de la convention-cadre et de ses principes fondateurs. Charge alors à la société civile de s'informer, de comprendre puis de proposer et d'initier des actions

\footnotetext{
${ }^{5}$ Projets pilotes de développement local (LDPP) est chargé de mettre en œuvre les principes de la Convention de Faro.

${ }^{6}$ Projets et Programmes-joints entre le Conseil de l’Europe et la Commission Européenne - Exemples en Europe du Sud-Est : Promotion de la diversité culturelle au Kosovo (PCDK) ou Stratégies urbaines dans les villes historiques (COMUS).

${ }^{7}$ Axes prioritaires : (1) la gestion de la diversité culturelle pour des sociétés cohésives et (2) l'amélioration du cadre et de la qualité de vie.

${ }^{8}$ Principes de Faro : (1) la citoyenneté s'appuie sur une communauté spécifiquement ancrée dans un territoire, (2) la cohésion sociale est refondée sur des modalités de participation et d'engagement et (3) la démocratie locale est renforcée par le développement des capacités d'action de la société civile.

${ }^{9}$ Neuf critères : (1) l'affirmation d'un groupe patrimonial, (2) l'émergence d'un consensus au sein de la communauté au sujet de la notion de patrimonialisation, (3) l'existence d'un territoire délimité et d'un imaginaire collectif qui lui est lié, (4) la capacité par le groupe à produire des récits de territoire et à susciter des récits de vie, (5) la présence et l'action de plusieurs passeurs, (6) l'appui d'acteurs politiques attentifs et bienveillants, (7) l'émergence d'un nouveau modèle économique, (8) l'affirmation d'un modèle participatif en appui de l'action publique et (9) l'ouverture sur des pratiques d' « empowerment ».
} 
patrimoniales de développement devant répondre aux enjeux politiques, éthiques et de gestion des « ressources collectives héritées du passé ". Les communautés patrimoniales (association, ONG, etc.) constituent alors la cellule « relais » devant impulser les dynamiques territoriales, bien qu'elles soient dotées de moyens souvent très limités pour identifier les valeurs liées à des aspects spécifiques du patrimoine culturel, fruit de la construction identitaire locale. Elles sont donc libres de s'activer sur tout type de ressources patrimoniales (monument, environnement, gastronomie, expression culturelle et orale, etc.) dès lors que ces dernières témoignent de la diversité du territoire par les savoirs et les productions des populations hérités du passé. Cette réappropriation stratégique (valeurs) et sectorielle (politique, économique, technologique, religieuse) des dynamiques territoriales post-conflits par les acteurs locaux répond, selon les orientations européennes, à la valorisation libre et collective (Applications Libres de Faro ou AFL) d'un patrimoine commun partagé dans l'espace géopolitique européen élargi. Par conséquent, toute initiative locale peut ainsi découler des principes de Faro, dès lors qu'elle se revendique AFL par ses acteurs auprès du CoE. Ce collectif d'initiatives nourrit ainsi la politique participative de l'organisation en tant que fondement culturel à la démocratie participative par la circulation des savoirs patrimoniaux, leur gestion intégrée et leur diffusion à diverses échelles géographiques (ville/local, nation/Kosovo, région/Balkans, Europe).

Comme nous l'avons vu dans la section précédente, les pays balkaniques étant désireux d'impulser une véritable dynamique quant à la ratification puis à la mise en œuvre de la Convention de Faro dans leur région, ils ont souhaité être associés à des actions de mise en œuvre des principes de la convention. Attentif à cette demande, le CoE a pu développer et mettre en place de nombreux projets spécifiques, dont un programme-joint (CoE/UE) consacré à la promotion de la diversité culturelle au Kosovo (PCDK) comme projet participatif en cohésion avec les valeurs du patrimoine culturel pour la société kosovare. Ce programme ${ }^{10}$ avait pour objectif d'encourager le développement lié à la planification durable du patrimoine et à sa gestion au Kosovo conformément aux normes et standards européens, en étroite collaboration avec la société civile, notamment les habitants, les ONG et les structures administratives locales (ministères et municipalités). Plusieurs villes kosovares ont ainsi été associées à ce programme, dont la petite localité agricole de Junik, située dans le district de Gjakovë, non loin de la capitale Pristina. Elle possède quelques vestiges architecturaux remarquables, dont des mosquées et des kullas, ou maisons fortifiées d'origine ottomane. Ces constructions domestiques, datant pour la plupart du $18^{\mathrm{e}}$, du $19^{\mathrm{e}}$ et du début du $20^{\mathrm{e}}$ siècles, ont été fortement endommagées durant les bombardements serbes, en raison des liens identitaires ancrés dans les racines culturelles et les pratiques séculaires des habitants. À la fin de la guerre, un plan de réhabilitation et de reconstruction de ces habitations traditionnelles a été adopté puis soutenu par la communauté internationale (COE, UNESCO) ainsi que par de nombreuses ONG européennes et américaines ${ }^{11}$, dans le but de préserver l'identité locale dans son paysage culturel tel qu'il était avant le conflit armé. La localité de Junik s'est peu à peu transformée en un laboratoire in situ de mise en œuvre des bonnes pratiques européennes de gestion intégrée du patrimoine (culture, nature), par la participation active de la société (réhabilitation), et elle est donc devenu un espace urbain susceptible de fournir des données qualitatives pertinentes pour mesurer l'application de certains principes de la Convention de Faro (Le Marec et Belaën, 2012). Dans ce contexte, le Bureau du CoE à Pristina a conduit une étude expérimentale par l'enquête ${ }^{12}$ en tant qu'observatoire in situ des principes de la Convention de Faro, afin d'analyser les dynamiques patrimoniales et sociétales (habitants) appliquées à un espace urbain reconnu comme patrimoine local par la population kosovare, à savoir la localité de Junik. La méthodologie empirique utilisée pour réaliser cette enquête qualitative est fondée sur un questionnaire et une série d’entretiens semi-directifs (en anglais et en albanais) auprès de 255 personnes, soit l'ensemble des habitants de plus de 15 ans présents dans le centre historique de Junik, découpé en six zones d'études. Cette enquête empirique avait pour objectif d'évaluer la circulation "top-down " des trois concepts ${ }^{13}$ fondateurs de la Convention de Faro, en les confrontant aux discours des acteurs locaux œuvrant à la défense du patrimoine. Nous ne présentons ici que quelques conclusions empiriques du rapport d'expert (Brianso, 2015), afin d'exposer en quoi les modalités de l'enquête favorisent l'émergence d'un espace de restitution par l'observatoire (Piponnier, 2014) dans un contexte social et patrimonial.

\footnotetext{
${ }^{10}$ PCDK I (2009-2012) et PCDK II (2012-2015).

${ }^{11} \mathrm{CHwB}, \mathrm{SIDA}, \mathrm{EAR}$, Packard Foudation, etc.

${ }^{12}$ Enquête (questionnaire et entretiens) réalisée en août 2014.

13 " communauté patrimoniale », " participation démocratique » et " patrimoine - patrimoine commun de l’Europe ».
} 
- La dimension sociale comme indicateur de qualité de vie : les acteurs locaux (habitants de Junik) considèrent le groupe social (famille, amis, etc.) comme un indicateur pertinent relatif à une qualité de vie satisfaisante. Les valeurs liées aux aspects spécifiques du patrimoine (culturel et naturel) ne sont considérées qu'en second plan.

- Le patrimoine naturel en tant que valeur culturelle: les habitants de de Junik semblent sensibles à l'environnement naturel en tant qu'espace de convivialité entre " communautés » et élément du patrimoine local, le plaçant au même niveau que le patrimoine culturel.

- Reconnaissance des éléments du patrimoine pour construire une identité nationale et européenne : les habitants de Junik considèrent que le patrimoine est un élément identitaire de premier plan, tant au niveau national que dans l'espace européen, dans une moindre mesure au niveau régional.

Les résultats montrent que les valeurs du patrimoine local (culture, nature, société) participent à la construction d'une identité européenne partagée, mais que les habitants de Junik n'adhèrent pas à la Convention de Faro, jugée trop abstraite. Pour autant, les habitants soulignent favorablement les actions européennes entreprises depuis la fin du conflit pour améliorer leur cadre de vie et ils souhaitent s'intégrer à un processus de développement socioéconomique grâce au tourisme. Les kullas réhabilitées sont par exemple transformées en gîte ou B\&B pour touristes, bien que des efforts restent encore à faire, tant dans les infrastructures touristiques (routes, équipements, etc.) que dans l'offre et les services (accueil, activités, etc.). Ces perspectives ont surtout été défendues par les femmes et les jeunes, qui souffrent du chômage et d'une organisation sociétale très patriarcale en milieu rural. Malgré une mise en œuvre mitigée sur le territoire kosovar, la Convention de Faro a permis d'élargir les points de vue des acteurs du territoire grâce à la mobilisation locale du tissu associatif. Cet observatoire in situ en construction constitue la trace tangible et visible du programme-joint financé par le CoE et offre un terrain de dialogue entre la recherche-action et la société civile.

\section{Conclusion}

La Convention de Faro constitue un standard européen original et innovant en matière patrimoniale par la rupture de posture quant aux valeurs sociales du patrimoine comme fondement éthique à l'initiative locale. Malgré ce choix politique ambitieux de voir émerger dans l'espace européen une " démocratie participative » favorisant la construction d'un "patrimoine commun de l'Europe ", la Convention de Faro ne connaît pas le même succès (ratification) auprès des États membres du Conseil de l'Europe que la "Convention du patrimoine mondial » de I'UNESCO (1972). Notons que les États membres de l'Europe historique sont restés prudents voire réticents à la ratification de ce texte (France), alors que d'autres, notamment à l'Est, y ont vu une opportunité politique d'intégration à l'Union européenne. II n'existe pas de véritable politique d'orientation des principes de Faro quant à leur mise en œuvre, mais plutôt une liberté d'interprétation des acteurs du territoire et d'initiatives collectives issues du Plan adopté en 2013. La société civile est donc libre de lire et d'interpréter la Convention de Faro (les AFL) selon ses besoins, même si des actions telles que les "Ateliers Faro » ou les "Ballades Faro » sont encouragées par le Conseil de l'Europe afin de créer une " communauté Faro " à partir d'un réseau de villes européennes - une " identité Faro » encore fragile, qui souligne les limites des comparaisons à des échelles territoriales diverses et concernant des espaces urbains très hétérogènes, présentant des dynamiques sociétales, patrimoniales et culturelles distinctes. Il serait ainsi délicat de comparer les initiatives Faro de villes comme Venise ou Marseille avec un territoire rural en reconstruction comme celui de Junik. Néanmoins, les principes éthiques fondés sur la participation active des habitants et sur des « communautés patrimoniales » constituent des valeurs communes, ce que nous avons essayé de présenter avec l'étude de cas au Kosovo. Par ailleurs, nous avons souligné le caractère exploratoire de l'enquête empirique, liée à la construction d'un observatoire en tant que « lien de production des connaissances en devenir " (Piponnier, 2014, p. 24) en collaboration avec la société civile. Enfin, notons que le Kosovo n'a pas encore stabilisé sa souveraineté au sein des instances politiques mondiales (ONU, CoE), malgré une indépendance proclamée en 2008. Il n'a donc pas une réelle existence politique auprès du CoE, sauf en référence à la "Résolution 1244 du Conseil de sécurité des Nations Unies et sans préjuger du statut du Kosovo », ce qui conditionne l'ensemble des actions menées au Kosovo à un angle exploratoire à partir de projets ou de programmes pilotes (Piponnier, 2012b) sous tutelle européenne (Commission Européenne, CoE). 


\section{Références}

Batisse, M. et Bolla, G. (2003). L'invention du « patrimoine mondial ». Paris : Association des anciens fonctionnaires de l'UNESCO (AAFU). (Les Cahiers d'Histoire 2, Club Histoire). http://whc.unesco.org/document/135225

Bortolotto, C. (2012). Nouveaux acteurs du patrimoine, nouvelles postures anthropologiques. Civilisations, 61(1), 139-146.

Brianso, I. (2015). PCDK Experience: Innovation in Democratic Participation. Strasbourg : Conseil de l'Europe.

Brianso, I. et Girault, Y. (2014). Innovations et enjeux éthiques des politiques environnementales et patrimoniales : UNESCO et Conseil de l'Europe. Revue internationale d'éthique sociétale et gouvernementale, 16(1), 17-37. (Enjeux éthiques des politiques publiques en matière d'environnement).

Chenevet, A. et Novello-Paglianti, N. (2015). L'invention de la Valeur Universelle Exceptionnelle de I'UNESCO. Paris : L'Harmattan.

Clanet, C. (1990). L'interculturel, introduction aux approches interculturelles en éducation et en sciences humaines. Toulouse : Presses Universitaires du Mirail.

Conseil de l'Europe (1949). Traité de Londres-Statut du Conseil de l'Europe. http://conventions.coe.int/Treaty/fr/Treaties/Html/001.htm

Conseil de l'Europe (1950). Convention européenne des droits de l'homme. http://www.echr.coe.int/Documents/Convention_FRA.pdf

Conseil de l’Europe (2005). Convention-cadre sur la valeur du patrimoine culturel pour la société. http://conventions.coe.int/Treaty/FR/Treaties/Html/199.htm

Conseil de l'Europe (2009). Déclaration-Programme régional pour le patrimoine culturel et naturel dans le sud-est de l'Europe. Dans R. Palmer (dir.), Le patrimoine et au-delà (p. 55-57). Strasbourg : Conseil de l'Europe.

Conseil de l'Europe (2014). Plan d'action pour la promotion de la Convention-Cadre de Faro. https://www.coe.int/t/dg4/cultureheritage/heritage/identities/AT-2014-510-Faro-AP_fr.pdf

De Sède-Marceau, M-H. et Moine, A. (2012). Les observatoires territoriaux. Une représentation collective du territoire. Communication et langages, 171, 55-65.

Demorgon, J., Müller, B. et Lipiansky, E.-M. (2003). Les dynamiques interculturelles pour l’Europe. Paris : Anthropos.

Demorgon, J. (1989). L'exploration interculturelle. Pour une pédagogie internationale. Paris : Armand Colin.

Filipovic, M. (2009). Pourquoi les États ratifient-ils les conventions? Le cas du Monténégro. Dans R. Palmer (dir.), Le patrimoine et au-delà (p. 51-53). Strasbourg : Conseil de l’Europe.

Goddard, S. (2009). Partenariats en faveur du patrimoine- Promouvoir la participation et la compréhension du public. Dans R. Palmer (dir.), Le patrimoine et au-delà (p. 149-158). Strasbourg : Conseil de l’Europe.

Labadi, S. (2013). UNESCO, Cultural heritage, and outstanding universal value : value-based analyses of the world heritage and intangible cultural heritage conventions. AltaMira Press.

Ladmiral, J. R. et Lipiansky, E.-M. (dir) (1989). La communication interculturelle, Paris, A. Colin.

Le Marec, J. et Belaën, F. (2012). La création d'un observatoire : que s'agit-il de représenter? Communication et langages, $171,67-80$.

Liévaux, P. (2009). La Convention de Faro, un outil original pour la construction et la gestion du patrimoine de l'Europe. Dans R. Palmer (dir.), Le patrimoine et au-delà (p. 49). Strasbourg : Conseil de l’Europe.

Matagne, P. (2009). La naissance de l'écologie. Paris : Ellipse.

Nations Unies. (1948). Déclaration universelle des droits de l'Homme. http://www.un.org/fr/documents/udhr/

Palmer, R. (2009). Préface. Dans R. Palmer (dir.), Le patrimoine et au-delà (p. 7-9). Strasbourg : Conseil de l’Europe. 
Paquette, J. (2012). Expertise et patrimoine autochtone : hybridation des savoirs et évolutions récentes des pratiques patrimoniales en Nouvelle-Zélande [en ligne]. Éthique publique, 14(1). http://ethique publique.revues.org/974

Piponnier, A. (2012a). Le projet. Une approche sociopragmatique d'un artefact communicationnel (Dossier d'habilitation à diriger des recherches, Paris 7-Diderot).

Piponnier, A. (2012b). Projet et observatoire. Une alliance historique et pragmatique. Communication et langages, $171,67-80$.

Piponnier, A. (2014). L'observatoire dans l'enquête [en ligne]. Sciences de la société, 92. http://sds.revues.org/946

UNESCO. (1966). Actes de la conférence générale de 1966, quatorzième session. Paris : Édition UNESCO. http://unesdoc.unesco.org/images/0011/001140/114048f.pdf

UNESCO. (1968). Évaluation du Projet majeur relatif à l'appréciation mutuelle des valeurs culturelles de l'Orient et de l'Occident. 1957-1966. Paris : Édition UNESCO. http://unesdoc.unesco.org/images/0013/001352/135250fo.pdf

UNESCO. (1972). Convention concernant la protection du patrimoine mondial, culturel et naturel. http://whc.unesco.org/archive/convention-fr.pdf 